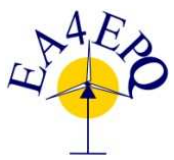

$18^{\text {th }}$ International Conference on Renewable Energies and Power Quality

(ICREPQ'20)

Granada (Spain), $1^{\text {st }}$ to $2^{\text {nd }}$ April 2020

Renewable Energy and Pomer Qualiny. Fournal (RE\&PQJ)

ISSN 2172-038 X, Volume No.18, June 2020

\title{
Wind generation in weak systems
}

\author{
L. Rouco, J. L. Zamora, and A. García-Cerrada \\ Universidad Pontificia Comillas \\ Alberto Aguilera, 23, 28015 Madrid, Spain
}

\begin{abstract}
Control systems of wind generators are usually designed neglecting the effect of the external grid (the impedance of the grid is much smaller than the impedance of the step-up transformers). Wind generator manufacturers are facing considerable challenges when connecting their machines to weak systems. A number of issues may arise. This paper classifies them and proposes a common approach to address them.
\end{abstract}

Keywords. Wind generation, weak systems, subsynchronous resonance, harmonic interaction, voltage stability, controller interaction.

\section{Introduction}

Control systems of wind generators are usually designed neglecting the effect of the external grid (the impedance of the grid is much smaller than the impedance of the step-up transformers). Wind generator manufacturers are facing considerable challenges when connecting their machines to weak systems. Weak systems are characterized by low short circuit ratio and/or low inertia. The question from the viewpoint of a wind generator manufacturer is how to squeeze the generator controls to maximize wind generation in a given point of connection.

A number of issues may arise in the systems with low short circuit ratio: subsynchronous oscillations, harmonic amplification, voltage stability and controller interaction. This paper describes models and methods developed in research projects for wind generation equipment manufacturers.

The paper is organized as follows. Section 2 reviews the features of weak systems. Section 3 summarizes phenomena arising in wide area systems. Section 4 proposes a set of test systems to investigate the phenomena of interest. Section 5 describes the developed simulation models. Section 6 details the study method. Section 7 provides some results of the investigation of one of the phenomena of interest. Section 8 provides the conclusions of the paper.

\section{Weak systems}

According to [1], an AC system is weak at the point of connection when any of the following conditions are met

- The short circuit ratio is low

- The mechanical inertia is low
The short circuit ratio (SCR) is the short circuit capacity $S_{s c}$ in per unit and also the inverse of the short circuit impedance $X_{s c}[2]$.

$$
S C R=\frac{S_{s c}(M V A)}{S_{B}(M V A)}=\frac{1}{X_{s c}(p u)}
$$

In case of remote wind generation, the short circuit impedance is very much related to the length and the nominal voltage of the connecting transmission system. As a matter of fact, the series reactance in per unit of a transmission line is proportional to its length 1 and to the inverse of the square of its nominal voltage $U_{n}$.

$$
X(p u)=\frac{X(\Omega)}{Z_{B}(\Omega)}=\frac{\omega L 1}{\frac{U_{n}^{2}}{S_{B}}}=\frac{2 \pi f_{0} \cdot 0.001 \cdot 1 \cdot S_{B}}{U_{n}^{2}}
$$

Frequency deviation is governed by the inertia of the rotating mass of generators and the response of the primary frequency regulation [3]. Fig. 1 depicts a model for investigating frequency dynamics which includes not only rotor and primary frequency regulation dynamics but also frequency load-shedding protection scheme.

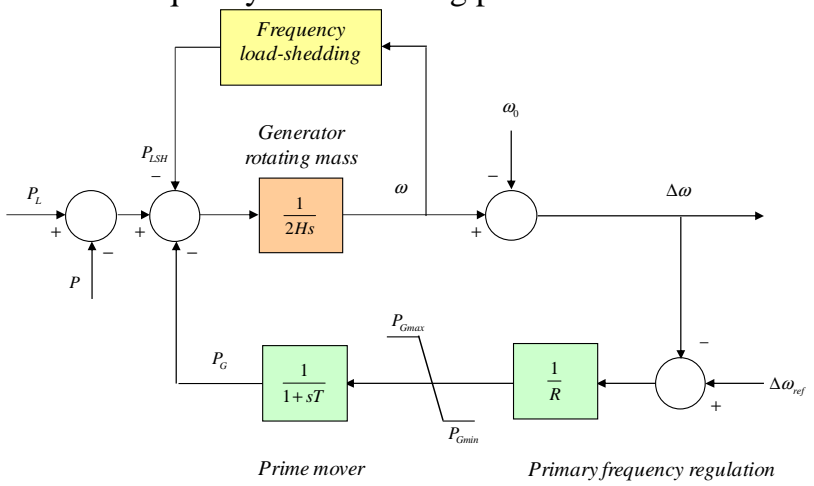

Fig. 1: Model for investigating frequency dynamics.

Assuming enough reserve and no activation of loadshedding, the steady state frequency deviation after a generation trip $P$ is

$$
\Delta \omega(\infty)=-R \cdot P
$$


It should be noted that the steady state frequency deviation does not depend on the inertia $H$ of the rotating masses. In contrast, the initial rate of change of frequency does

$$
\left.\frac{d \Delta \omega}{d t}\right|_{t=0^{+}}=\frac{-P}{2 H}
$$

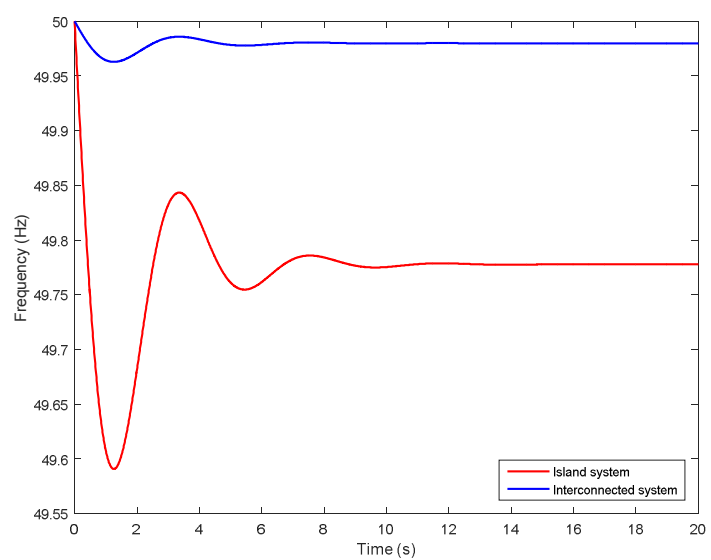

Fig. 2: Comparison of the frequency excursion in a large interconnected system and in an island system.

Fig. 2 compares the frequency excursion in a large interconnected system and in an island system. A 3,000 MW generation loss is considered in a large interconnected system $(300,000 \mathrm{MW}$ system) while a 100 MW generation loss is considered in an island system (1,000 MW system). It must be noted that frequency excursions in large interconnected systems is very much small assuming ideal performance of primary frequency regulation. Moreover, in both cases the minimum frequency (frequency nadir) is well above the typical setting of underfrequency load shedding schemes (49 $\mathrm{Hz})$.

This paper will be focused on weak systems arising in large interconnected systems that exhibit low short circuit ratio.

\section{Phenomena in low short circuit ratio systems}

Four phenomena can arise in low short circuit ratio systems:

- Subsynchronous oscillations.

- Harmonic amplification.

- Voltage stability.

- Controller interaction.

Subsynchronous resonance may occur in turbogenerators (synchronous generators driven by steam turbines) connected to the grid through series compensated lines (see Fig. 3) [4]. The natural oscillation of the series LC circuit is seen by the synchronous generator rotor as two natural oscillations of subsynchronous and supersynchronous frequencies respectively according to where

$$
f_{1,2}=f_{0} \pm f_{n}
$$

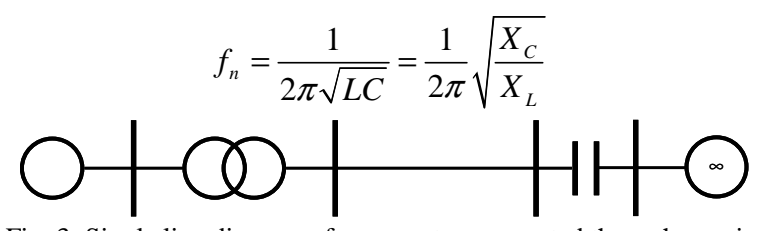

Fig. 3: Single line diagram of a generator connected through a series compensated line to an infinite bus.

The frequency of the subsynchronous oscillation may be close to a torsional frequency of the rotor making it unstable. Fig. 4 depicts the multi-mass model of a turbogenerator that exhibits torsional oscillations among turbogenerator masses. In addition, the subsynchronous frequency may be unstable itself resulting in induction generator behavior. Subsynchronous oscillations have occurred in wind farms connected through series compensated lines ([5], [8]) resulting in electrical failures in contrasts to the mechanical issues that arise in subsynchronous resonance of synchronous generators.

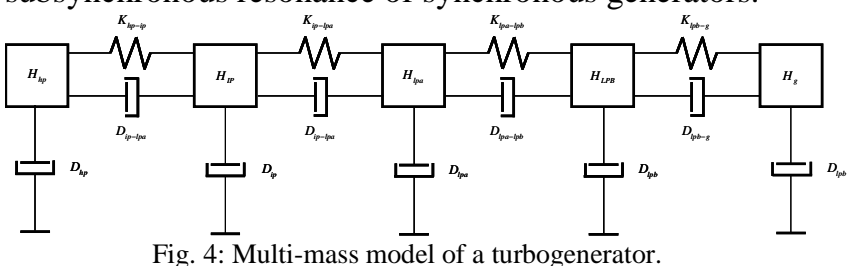

Harmonic amplification may occur due to a shunt capacitor bank installation (see Fig. 5). As a matter of fact, large harmonic voltages can arise due to harmonic currents when the equivalent impedance seen from the harmonic sources (the parallel of grid impedance and the capacitor bank reactance) is very large. The equivalent impedance would be infinite for a harmonic of order

$$
h=\sqrt{\frac{X_{C}}{X_{L}}}=\frac{1}{\sqrt{X_{L} B_{C}}}=\sqrt{\frac{S_{s c}}{Q_{C}}}
$$

where $S_{s c}$ is the short circuit capacity at the point of common coupling and $Q_{C}$ is the rated reactive power supplied by the shunt capacitor bank.

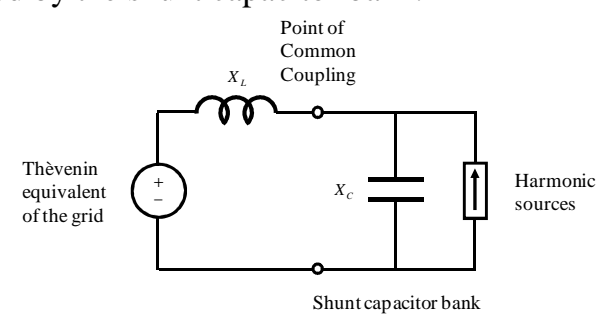

Fig. 5: Equivalent circuit to study harmonic amplification due to a shunt capacitor bank.

Fig. 6 depicts the grid reactance, the capacitor reactance and the equivalent reactance as a function of the harmonic order. Wind generator type 3 and type 4 are harmonic sources and wind farms make use of shunt capacitor banks to comply with the reactive power 
requirements. Hence, wind farms are subject to harmonic amplification [9].

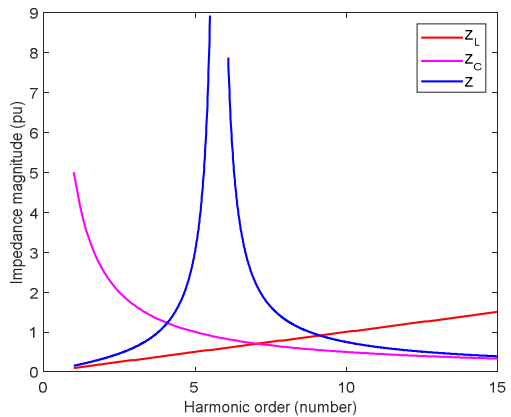

Fig. 6: Grid reactance, capacitor reactance and equivalent reactance as a function of the harmonic order.

Voltage stability is concerned with the ability of generators to supply the loads at admissible voltage ranges [10]. Voltage stability is related the maximum power transfer capability of a circuit. When a generator is connected to an infinite bus (Fig. 7), the maximum power transfer capability is provided by the maximum of the power-angle characteristic (Fig. 8) which is the inverse of the line reactance assuming rated voltages.

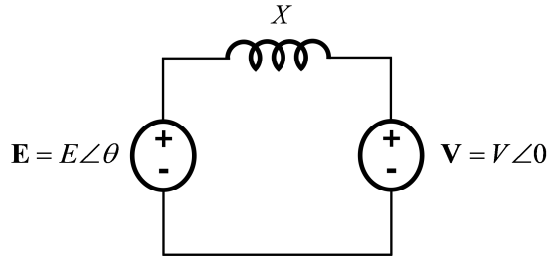

Fig. 7: Equivalent circuit of a generator connected to an infinite bus.

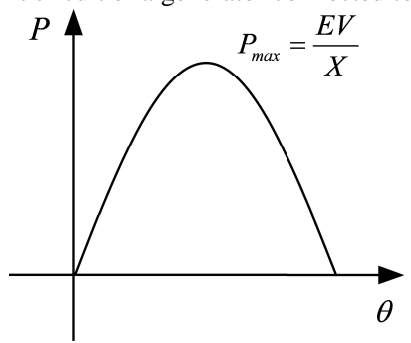

Fig. 8: Power - angle characteristic of a generator connected to an infinite bus.

In contrast, when a generator is feeding a load (Fig. 9), the maximum power transfer capability is provided by the PV curves (Fig. 10). It must be noted that for a unity power factor load the maximum power transfer capability in 0.5 the maximum power transfer when the generator is connected to an infinite bus.

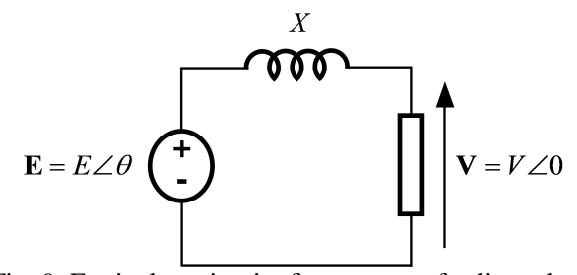

Fig. 9: Equivalent circuit of a generator feeding a load.

Voltage stability may also constraint the ability of wind generation to deliver its output through long transmission systems ([12]-[14]).
Statcoms are frequently are installed at the medium voltage bus of wind farms for voltage and reactive power control as shown in Fig. 11. In such situations, interaction between wind generators controls and Stacom controls may occur.

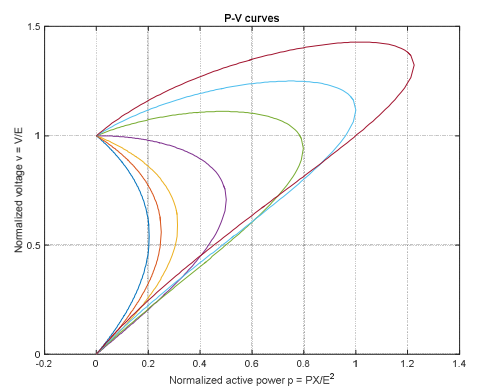

Fig. 10: PV curves for several load power factors.

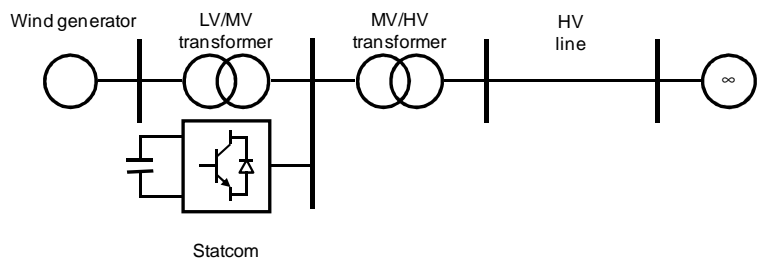

Fig. 11: Example of controller interaction.

\section{Test cases}

The study of each phenomenon under investigation requires the use of a specific test case. Fig. 12 shows the proposed test case for investigating subsynchronous oscillations. A wind generator is connected to an infinite bus through a series RLC circuit. The reactance of the series capacitor is usually expressed as a factor of the reactance of the series inductor.

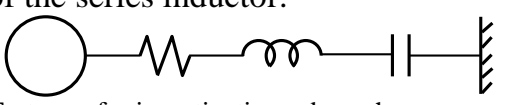

Fig. 12: Test case for investigating subsynchronous oscillations.

Fig. 13 shows the test case for investigating harmonic amplification. A wind generator is connected to an infinite bus through a RLCRL circuit.

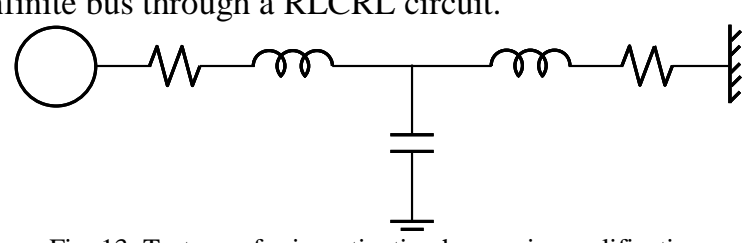

Fig. 13: Test case for investigating harmonic amplification.

Fig. 14 shows the test case the test case for investigating voltage stability. A wind generator is connected to an infinite bus through a RL circuit.

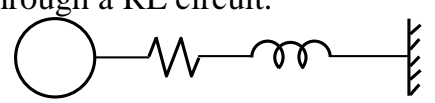

Fig. 14: Test case for investigating voltage stability.

Fig. 15 shows the test case the test case for investigating controller interaction. A wind generator is connected to an infinite bus through a RLRL circuit. 


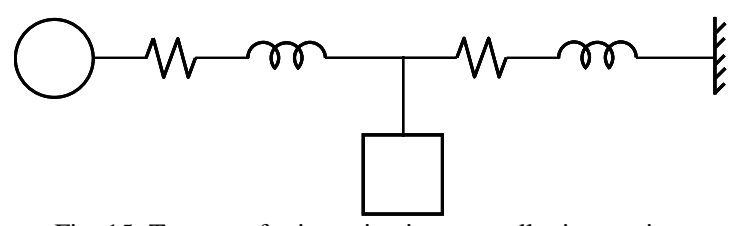

Fig. 15: Test case for investigating controller interaction.

\section{Simulation models}

There are three modeling options when developing simulation models for investigating the phenomena of interets:

- Electromagnetic transients including switches of power electronic converters.

- Electromagnetic transients with ideal models of power electronic converters.

- Electromechanical transients.

We have chosen an electromagnetic model with ideal models of power electronic convertes since they allow not only to perform time domain simulations but also eigenvalue and frequency domain analysis of the linearized model. In addition, we have adopted a modular representation. A module is used to represent the wind generator and one or several modules to represent the grid.

Fig. 16 shows the Matlab/Simulink model for investigating subsynchronous oscillations. There are three components: the wind generator (solid box), the RLC grid (dashed box) and the point of common coupling.

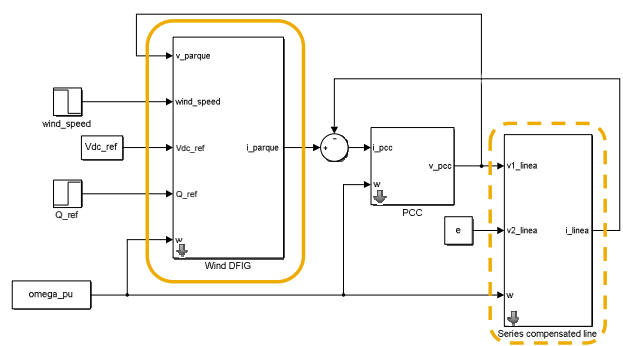

Fig. 16: Model for investigating subsynchronous oscillations

Fig. 17 shows the Matlab/Simulink model for investigating harmonic amplification. There are three components: the wind generator (solid box), the RLCRL grid (dashed box) and the point of common coupling.

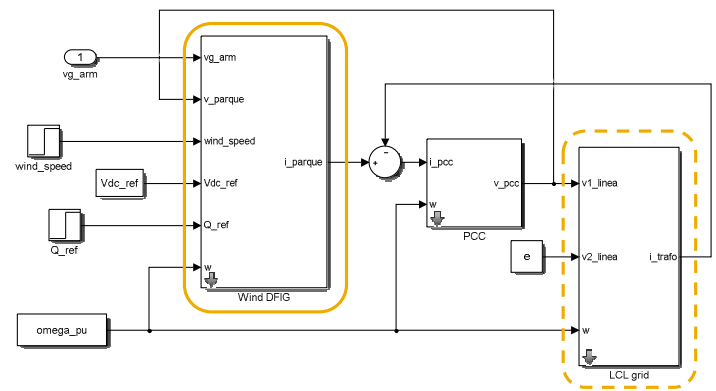

Fig. 17: Model for investigating harmonic amplification.
Fig. 18 shows the Matlab/Simulink model for investigating voltage stability. There are three components: the wind generator (solid box), the RL grid (dashed box) and the point of common coupling.

Fig. 19 shows the Matlab/Simulink model for investigating controller interaction. There are six components the wind generator (solid box), the Stacom (solid box), two RL grids (dashed box) and two points of coupling.

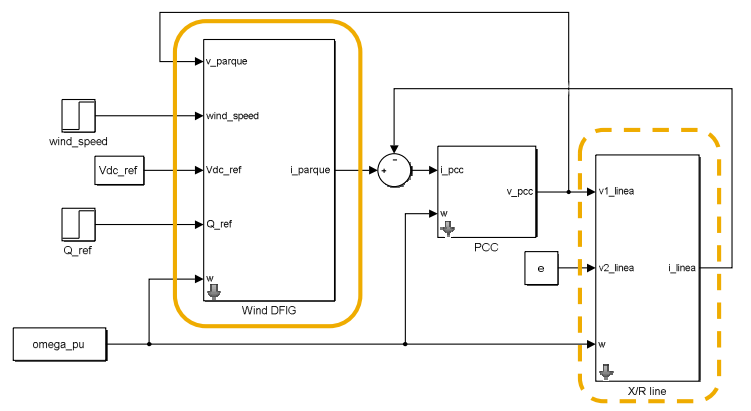

Fig. 18: Model for investigating voltage stability.

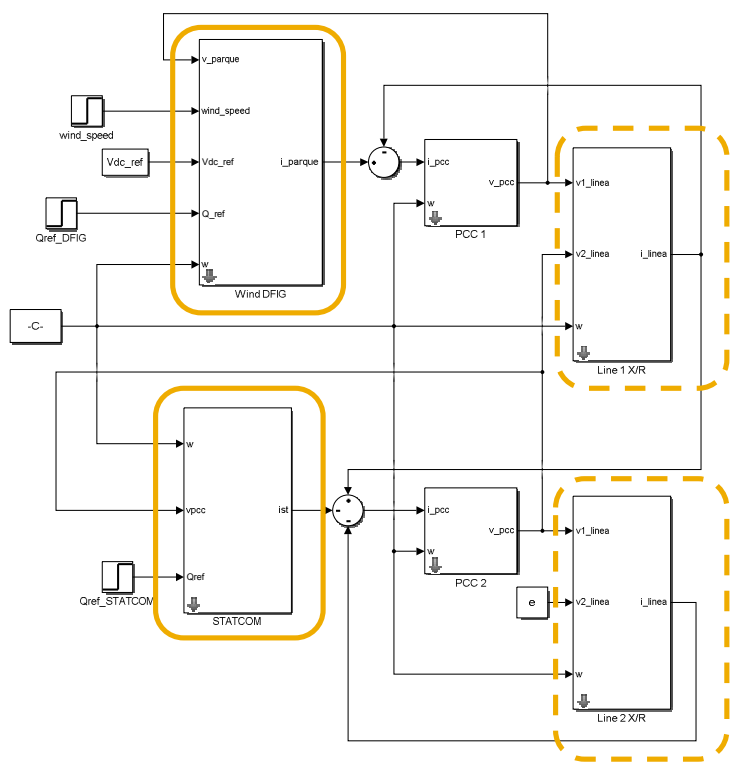

Fig. 19: Model for investigating controller interaction.

\section{Study method}

Simulation models presented in the previous section results in set of non-linear differential equations

$$
\begin{aligned}
& \mathbf{k}=\mathbf{F}(\mathbf{x}, \mathbf{u}, \boldsymbol{\eta}) \\
& \mathbf{y}=\mathbf{G}(\mathbf{x}, \mathbf{u}, \boldsymbol{\eta})
\end{aligned}
$$

where $\mathbf{x}$ is the vector of state variables, $\mathbf{u}$ is the vector of input variables, $\mathbf{y}$ is the vector of output variables, $\boldsymbol{\eta}$ is a vector of parameters and $\mathbf{F}$ and $\mathbf{G}$ are vectors of non-lineas functions. An operating point $\left(\mathbf{x}_{0}, \mathbf{u}_{0}\right)$ is an equilibrium point when

$$
\mathbf{0}=\mathbf{F}\left(\mathbf{x}_{0}, \mathbf{u}_{0}, \boldsymbol{\eta}\right)
$$


If equations (2) are linearized around the $\left(\mathbf{x}_{0}, \mathbf{u}_{0}\right)$ equilibrium point, they result in

$$
\begin{aligned}
& \Delta \mathbf{k}=\left.\frac{\partial \mathbf{F}(\mathbf{x}, \mathbf{u}, \boldsymbol{\eta})}{\partial \mathbf{x}}\right|_{\mathbf{x}_{0}, \mathbf{u}_{0}} \Delta \mathbf{x}+\left.\frac{\partial \mathbf{F}(\mathbf{x}, \mathbf{u}, \boldsymbol{\eta})}{\partial \mathbf{u}}\right|_{\mathbf{x}_{0}, \mathbf{u}_{0}} \Delta \mathbf{u} \\
& \Delta \mathbf{y}=\left.\frac{\partial \mathbf{G}(\mathbf{x}, \mathbf{u}, \boldsymbol{\eta})}{\partial \mathbf{x}}\right|_{\mathbf{x}_{0}, \mathbf{u}_{0}} \Delta \mathbf{x}+\left.\frac{\partial \mathbf{G}(\mathbf{x}, \mathbf{u}, \boldsymbol{\eta})}{\partial \mathbf{u}}\right|_{\mathbf{x}_{0}, \mathbf{u}_{0}} \Delta \mathbf{u}
\end{aligned}
$$

Or in a more compact form

$$
\begin{aligned}
& \Delta \mathbb{R}=\mathbf{A} \Delta \mathbf{x}+\mathbf{B} \Delta \mathbf{u} \\
& \Delta \mathbf{y}=\mathbf{C} \Delta \mathbf{x}+\mathbf{D} \Delta \mathbf{u}
\end{aligned}
$$

The stability of the linear system (3) can be assessed by inspecting the solution to the undriven system (also called homogeneous solution)

$$
\Delta \mathbf{x}(t)=e^{\mathbf{A} t} \Delta \mathbf{x}(0)=\sum_{i=1}^{N} \mathbf{v}_{i} e^{\lambda_{i} t}\left[\mathbf{w}_{i}^{T} \Delta \mathbf{x}(0)\right]
$$

where $\mathbf{v}_{i}$ and $\mathbf{w}_{i}$ are respectively the right and left eigenvectors corresponding to the eigenvalue $\lambda_{i}$ of the state matrix A are defined according to

$$
\begin{gathered}
\mathbf{A} \mathbf{v}_{i}=\mathbf{v}_{i} \lambda_{i} \\
\mathbf{w}_{i}^{T} \mathbf{A}=\lambda_{i} \mathbf{w}_{i}^{T} \\
\mathbf{w}_{i}^{T} \mathbf{v}_{i}=1
\end{gathered}
$$

Hence, the inspection of (4) allows to drawn the following results

- The solution is the sum of $\mathrm{N}$ components corresponding, each corresponding to each eigenvalue.

- A real negative (positive) eigenvalue means an exponentially decreasing (increasing) monotonic behaviour (see Fig. 20).

- A complex eigenvalue of negative (positive) real part means an exponentially decreasing (increasing) oscillatory behaviour (see Fig. 20).

- The components of the right eigenvectors inform about the relative activity of a variable in an eigenvalue.

- The components of the left eigenvectors weight the initial conditions in an eigenvalue.

The relationships between eigenvalues and state variables can be further investigated putting together the information provided by right and left eigenvectors. The participation factor of the $j$-th variable in the $i$-th mode is defined as [15]

$$
p_{j i}=w_{j i} v_{j i}
$$

In addition to the eigenvalue analysis, the frequency response may be useful. The frequency response of the transfer function between the $l$-th input and the $k$-th output can be calculated as

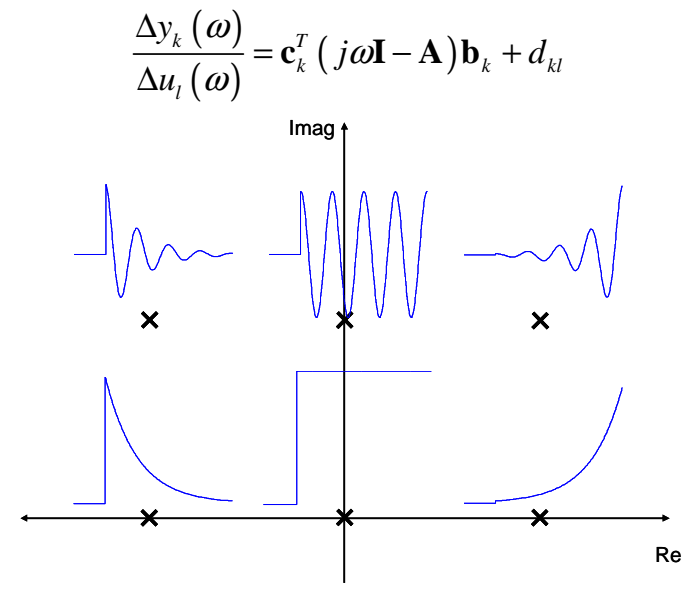

Fig. 20: Stability and eigenvalue location in the complex plane.

Fig. 21 provides an overview of the study method. The first step consists of calculating the equilibrium point. Non-linear time domain simulation can be performed. In addition, the linear model model can be calculated and then eigenvalue analysis and frequency response calculations can be subsequently performed.

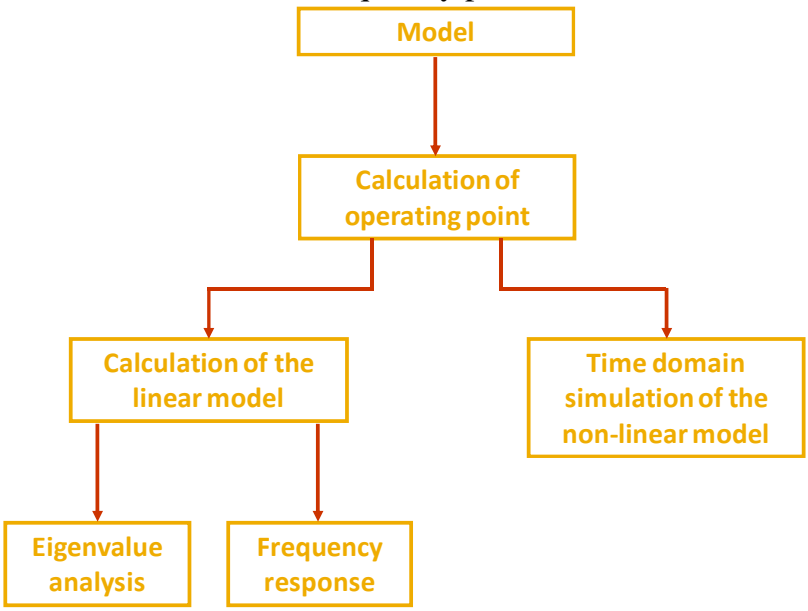

Fig. 21: Overview of the study method.

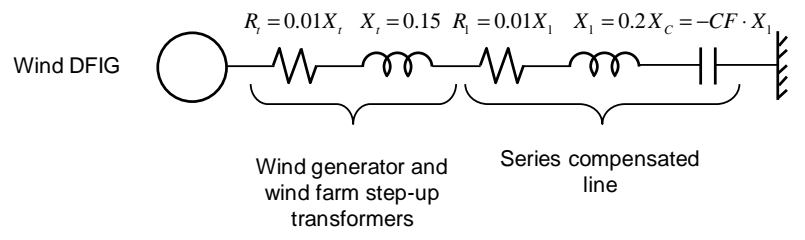

All parameters are in per unit of the MVA base of the wind generator

Fig. 22: Details of the test case for investigating subsynchronous oscillations of a wind DFIG.

\section{Illustrative results}

The models, methods and tools are illustrated investigating subsynchronous oscillations of a wind doubly fed induction generator (DFIG) connected through a series compensated line according to the test case of Fig. 22. The reactance of the series capacitor is expressed in terms of the compensation factor and the series reactance of the compensated line. Fig. 23 contains the parameters of the wind DFIG generator. 


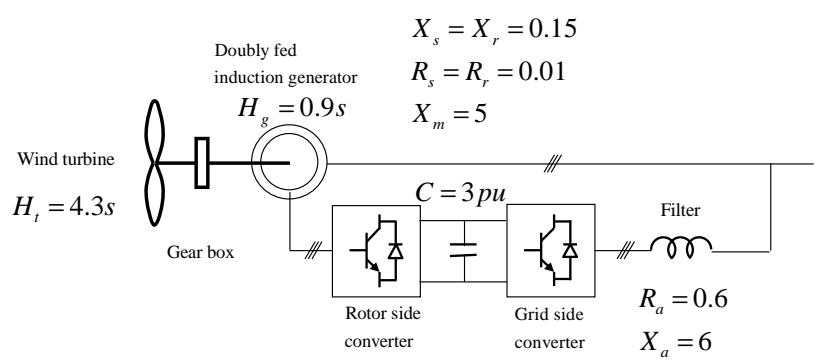

Fig. 23: Wind DFIG parameters.

An estimation of the natural frequency of the series LC circuit in case of $20 \%$ compensation factor

$$
\begin{aligned}
& f_{n}=f_{0} \sqrt{\frac{X_{C}}{X_{L}}}=f_{0} \sqrt{\frac{F C \cdot X_{1}}{X_{s}+X_{r}+X_{t}+X_{1}}} \\
& =50 \sqrt{\frac{0.2 \cdot 0.2}{0.15+0.15+0.15+0.2}}=12.4 \mathrm{~Hz}
\end{aligned}
$$

Hence, the frequencies of the supersychronous and subsynchronous oscillations are respectively

$$
\begin{aligned}
& f_{0}+f_{n}=62.4 \mathrm{~Hz} \\
& f_{0}-f_{n}=37.6 \mathrm{~Hz}
\end{aligned}
$$

The impact of the compensation factor on the stability is the subsynchronous oscillations is studied firstly. Fig. 24 shows the root locus as the compensation factor increases. A very interesting result arises: the supersynchronous mode is stable no matter the compensation factor whereas the subsynchronous mode becomes unstable for high compensation factors.

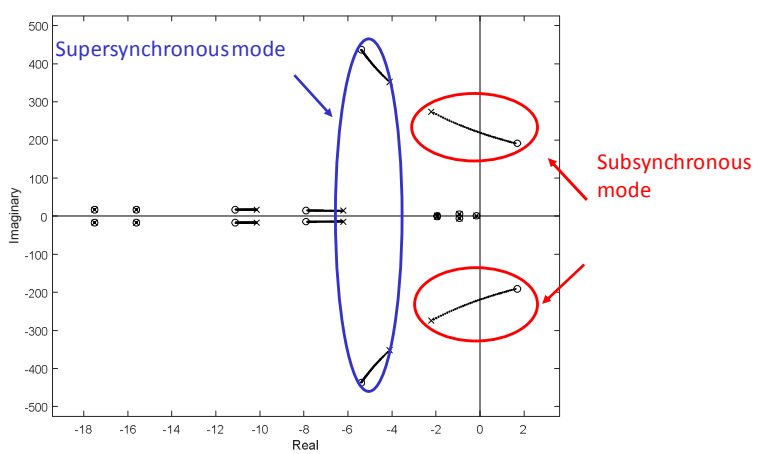

Fig. 24: Root locus as the compensation factor increases.

Moreover, Fig. 25 displays the damping of the subsysnchronous mode as a function of the compensation factor and the wind speed. It shows that the damping of the subsynchronous mode does not depend on the wind speed.

The compensation factor of the series compensated line the bandwidth of the wind DFIG controllers is worth to be investigated as well. Fig. 26 shows the variation of the damping of the subsychronous mode as function of the bandwidth of the controllers of rotor side converter (RSC) and the grid side converter (GSC). The damping of the subsynchronous model is affected by the bandwidth of the rotor side converter regulators. The bandwidth of the regulators of the grid side converter does not affect the damping of the subsynchronous mode In case of a compensation factor of $30 \%$ and a wind speed of $10 \mathrm{~m} / \mathrm{s}$, the mode becomes unstable if the bandwidth is 32.5 $\mathrm{rad} / \mathrm{s}$.

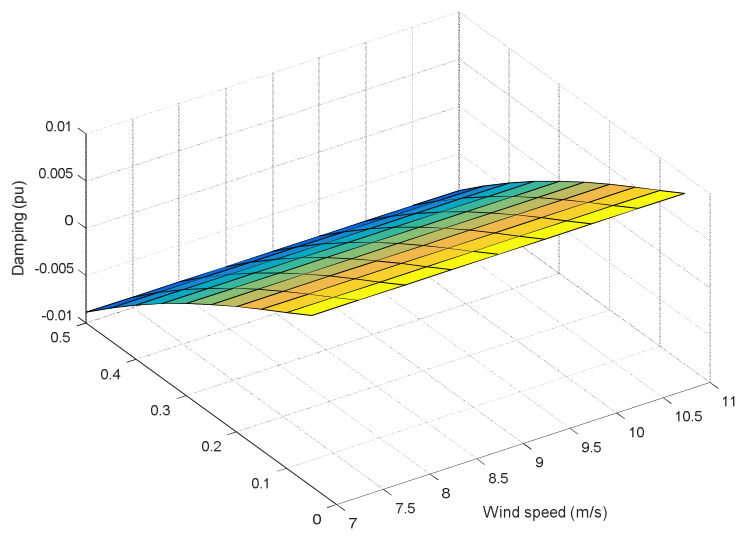

Compensation factor (pu)

Fig. 25: Damping of the subsychronous mode as function of the compensation factor and the wind speed.

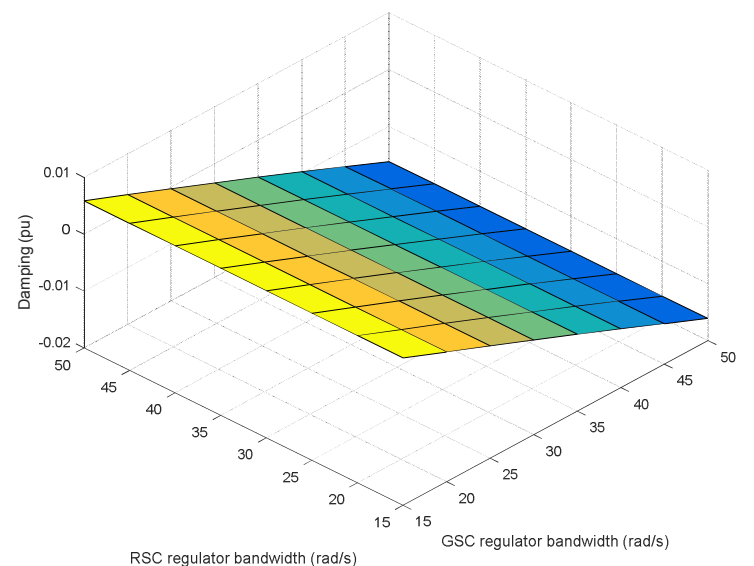

Fig. 26: Damping of the subsychronous mode as function of the bandwidth of the controllers of rotor side converter (RSC) and the grid side converter (GSC).

\section{Conclusions}

This paper has identified a number of phenomena arising in the connection of wind generation to weak systems characterized by low short circuit ratio. The paper has proposed simulation models and analysis methods to investigate them. The paper has also provided a detailed report on the investigation on the stability of subsynchronous oscillations arising in the connection of wind DFIG generators through series compensated lines. The findings of the study can be summarized as follows:

- The stability of subsycnrhonous ocillations is determined by the subsynchronous mode.

- The damping of the subsynchronous mode depends on the line compensation factor. It does not depend on the wind speed.

- The damping of the subsynchronous mode depends on the bandwidth of the regulators of the rotor side converter. Higher bandwidth results in undamped mode. 
- The bandwidth of the regulators of the grid side converter does not affect the damping of the subsynchronous mode.

\section{References}

[1] IEEE, "IEEE Guide for Planning DC Links Terminating at AC Locations Having Low Short-Circuit Capacities", IEEE Std. 12041997 ,

[2] O. I. Elgerd, "Electric Energy Systems Theory: An Introduction", Second Edition, Mc Graw Hill, 1982.

[3] L. Sigrist, E. Lobato, F. M. Echavarre, I. Egido, L. Rouco, "Island Power Systems", CRC Press, 2016.

[4] P. M. Anderson, B. L. Agrawal y J. E. Van Ness, "Subsynchronous Resonance in Power Systems", IEEE Press, 1989.

[5] J. Adams, C. Carter, C.; H. Shun-Hsien, "ERCOT Experience with Sub-synchronous Control Interaction and proposed remediation" 2012 IEEE PES Transmission and Distribution Conference and Exposition (T\&D), Orlando, Florida, May 7-10, 2012, Paper No. 2012TD0609.

[6] J. Adams, V. A. Pappu, A. Dixit, "ERCOT Experience Screening for Sub-Synchronous Control Interaction in the Vicinity of Series Capacitor Banks", 2012 IEEE Power and Energy Society General Meeting, San Diego, California, July 22-27, 2012, Paper 2012GM1447.

[7] F. Lingling, Z. Chanxia, M. Zhixin, H. Minqiang, "Modal Analysis of a DFIG-Based Wind Farm Interfaced With a Series Compensated Network", IEEE Transactions on Energy Conversion, Vol. 26, No. 4, 2011, pp. 1010 - 1020.

[8] F. Lingling, R. Kavasseri, M. L. Zhixin Lee, Z. Chanxia, "Modeling of DFIG-Based Wind Farms for SSR Analysis", IEEE Transactions on Power Delivery, Vol.: 25, No. 4, December 2010, pp. 2073-2082

[9] S. A. Papathanassiou, M. P. Papadopoulos, "Harmonic Analysis in a Power System with Wind Generation", IEEE Transactions on Power Delivery, Vol. 21, No. 4, October 2006, pp. 2006 - 2016.

[10] T. Van Cutsem, C. Vournas, "Voltage Stability of Electric Power Systems," Kluwer Academic Publishers, 1998.

[11] V. Diedrichs, A. Beekmann, K. Busker, S. Nikolai, S. Adloff, "Control of wind power plants utilizing voltage source converter in high impedance grids", 2012 IEEE Power and Energy Society General Meeting, San Diego, California, July 22-27, 2012, Paper No. 2012GM1467.

[12] J. W. Feltes, B. S. Fernandes, "Wind turbine generator dynamic performance with weak transmission grids", 2012 IEEE Power and Energy Society General Meeting, San Diego, California, July 22-27, 2012, Paper No. 2012GM1828.

[13] H. Chong; A. Q. Huang, M.E. Baran, S. Bhattacharya, W. Litzenberger, L. Anderson, A. L. Johnson, A.-A. Edris, "STATCOM Impact Study on the Integration of a Large Wind Farm into a Weak Loop Power System", IEEE Transactions on Energy Conversion, Vol. 23, No. 1, March 2008, pp. 226 - 233

[14] N.R. Ullah, T. Thiringer, D. Karlsson, "Voltage and Transient Stability Support by Wind Farms Complying With the E.ON Netz Grid Code", IEEE Transactions on Power Systems, Vol. 22, No. 4, November 2007, pp. $1647-1656$.

[15] I. J. Pérez-Arriaga, G. C. Verghese, F. C. Schweppe, "Selective Modal Analysis with Applications to Electric Power Systems. Part I: Heuristic Introduction. Part II: The Dynamic Stability Problem". IEEE Transactions on Power Apparatus and Systems, Vol. PAS101, No. 9, September 1982, pp. 3117-3134.

\section{Acknowledgements}

This work has been supported the Spanish Ministry of Science through project IPT-2011-1142-920000. The project has also involved Ingeteam Technology,S.A, Ingeteam Energy, S.A. and Universidad del País Vasco. 